\title{
Mass and position determination of attached particles on cantilever based mass sensors
}

Dohn, Søren; Svendsen, Winnie Edith; Boisen, Anja; Hansen, Ole

Published in:

Review of Scientific Instruments

Link to article, DOI:

$10.1063 / 1.2804074$

Publication date:

2007

Document Version

Publisher's PDF, also known as Version of record

Link back to DTU Orbit

Citation (APA):

Dohn, S., Svendsen, W. E., Boisen, A., \& Hansen, O. (2007). Mass and position determination of attached particles on cantilever based mass sensors. Review of Scientific Instruments, 78(10), 103303.

https://doi.org/10.1063/1.2804074

\section{General rights}

Copyright and moral rights for the publications made accessible in the public portal are retained by the authors and/or other copyright owners and it is a condition of accessing publications that users recognise and abide by the legal requirements associated with these rights.

- Users may download and print one copy of any publication from the public portal for the purpose of private study or research.

- You may not further distribute the material or use it for any profit-making activity or commercial gain

- You may freely distribute the URL identifying the publication in the public portal 


\title{
Mass and position determination of attached particles on cantilever based mass sensors
}

\author{
S. Dohn, ${ }^{a}$ W. Svendsen, and A. Boisen \\ MIC, Department of Micro- and Nanotechnology, NanoDTU, Technical University of Denmark, Building \\ 345E, DK-2800 Lyngby, Denmark \\ O. Hansen \\ Danish National Research Foundation's Center for Individual Nanoparticle Functionality (CINF), \\ Department of Micro- and Nanotechnology, NanoDTU, Technical University of Denmark, Building 345E, \\ DK-2800 Lyngby, Denmark
}

(Received 26 April 2007; accepted 6 October 2007; published online 31 October 2007)

\begin{abstract}
An analytical expression relating mass and position of a particle attached on a cantilever to the resulting change in cantilever resonant frequency is derived. Theoretically, the position and mass of the attached particle can be deduced by combining measured resonant frequencies of several bending modes. This finding is verified experimentally using a microscale cantilever with and without an attached gold bead. The resonant frequencies of several bending modes are measured as a function of the bead position. The bead mass and position calculated from the measured resonant frequencies are in good agreement with the expected mass and the position measured.
\end{abstract}

(c) 2007 American Institute of Physics. [DOI: 10.1063/1.2804074]

In the recent years a wealth of nanoelectromechanical systems has emerged, and one of the obvious applications of these is in mass sensing. ${ }^{1}$ Since the method was proposed in $1995,{ }^{2,3}$ cantilever based mass sensors have developed into promising candidates for ultrasensitive mass sensing, and recently systems capable of detecting masses in the atto- and zeptogram $\left(10^{-18}-10^{-21} \mathrm{~g}\right)$ ranges have been reported. ${ }^{4,5}$ The ultimate goal of single molecule detection capabilities of cantilever based mass sensors thereby seems to be within reach. ${ }^{6}$

Previous models for cantilever mass sensors are evaluated assuming either that the added molecules are distributed in a homogeneous layer covering all of the cantilever ${ }^{5}$ or that they are positioned as a point mass at the tip of the cantilever. The mass response of the cantilever, however, will change with the actual position of the added mass since the vibration velocity of the cantilever surface varies with position; ${ }^{8}$ thus the position of the added mass must be known in order to fulfill the vision of single molecule sensitivity.

It follows that a method that allows simultaneous detection of the mass and position of the added mass is needed. Such a method could also prove very useful in the emerging field of hollow cantilevers ${ }^{9}$ in which target molecules flow through the cantilever and, in some approaches, adsorb on the inner surface at a random position. The hollow cantilever will not allow for visual detection of the position of the molecules, thus a method for revealing both position and mass of the adsorbates would be beneficial.

Here, we derive relations between the resonant frequencies of a mass loaded cantilever and position and mass of the added molecules. These relations allow the mass and position of the added mass to be determined even without $a$ priori knowledge about the position. The method relies on measurements of the native as well as the mass loaded cantilever resonant frequencies for several vibration modes. The

${ }^{\text {a)} E l e c t r o n i c ~ m a i l: ~ s d @ m i c . d t u . d k ~}$ method is verified using experimental data $^{8}$ and proves to resolve both position and mass with high accuracy.

Consider a cantilever with the mass $m_{0}$ loaded by a point mass $\Delta m$ positioned at $z_{\Delta m}$ (Fig. 1). The displacement function of the vibrating beam is $W_{n}(z, t)=a_{n} U_{n}(z) e^{-i \omega_{n} t}$, where $n$ denotes the modal number, $\omega_{n}$ is the resonant frequency, $U_{n}(z)$ is the time independent mode shape, and $a_{n}$ is the modal amplitude at mode $n$. The mode shape for a clampedfree beam (cantilever) takes the form ${ }^{10}$

$$
U_{n}(z)=A_{n}\left(\cos \kappa_{n} z-\cosh \kappa_{n} z\right)+B_{n}\left(\sin \kappa_{n} z-\sinh \kappa_{n} z\right),
$$

where the modal wavenumbers $\kappa_{n}$ are solutions to $\cos \left(\kappa_{n} L\right) \cosh \left(\kappa_{n} L\right)=-1$, and the mode coefficients fulfill $A_{n} / B_{n}=\left(\cos \kappa_{n} L+\cosh \kappa_{n} L\right) /\left(\sin \kappa_{n} L-\sinh \kappa_{n} L\right)$. Numerical values of the first modal wavenumbers and corresponding mode coefficients are $\kappa_{n} L=1.875,4.694,7.855,10.996, \ldots$ and $A_{n} / B_{n}=-1.362,-0.982,-1.001,-1.000, \ldots$ Below, normalized mode shapes, $\int_{0}^{L} U_{n}^{2}(z) d x=L$, are assumed.

If the mass load is much less than the cantilever mass, $\Delta m \ll m_{0}$, the cantilever mode shape will not change significantly; thus the resonant frequency of such a system can be accurately estimated using an energy approach and the Rayleigh-Ritz theorem. According to the Rayleigh-Ritz theorem the time average kinetic energy $E_{\mathrm{kjin}}$ equals the time average strain energy $E_{\text {strain }}$ at resonance. ${ }^{\text {Th }}$ Thus, for a cantilever with an attached point mass, $E_{\text {strain }}=E_{\text {kin }}+E_{\text {kin, } \Delta m}$, where $E_{\text {kin, } \Delta m}$ is the kinetic energy due to the point mass. The kinetic energy of the cantilever is

$$
\begin{aligned}
E_{\mathrm{kin}} & =\int_{V} \frac{1}{2} \rho \omega_{n, \Delta m}^{2} a_{n}^{2} U_{n}^{2}(z) d V \\
& =\frac{1}{2} w h \rho a_{n}^{2} \omega_{n, \Delta m}^{2} \int_{0}^{L} U_{n}^{2}(z) d x=\frac{1}{2} m_{0} a_{n}^{2} \omega_{n, \Delta m}^{2},
\end{aligned}
$$

where $\rho$ is the average mass density of the cantilever. The kinetic energy due to the added point mass at $z_{\Delta m}$ is 


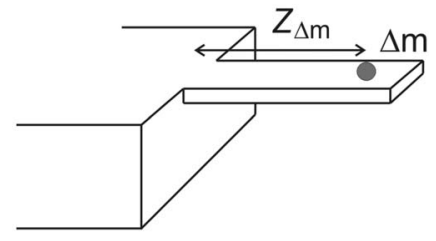

FIG. 1. Schematic of a cantilever with a single bead of mass $\Delta m$ positioned at $z_{\Delta m}$.

$$
E_{\mathrm{kin}, \Delta m}=\frac{1}{2} \Delta m \omega_{n, \Delta m}^{2} a_{n}^{2} U_{n}^{2}\left(z_{\Delta m}\right) .
$$

Since the mode shape is assumed unchanged by the attached point mass, the strain energy is approximately equal to the kinetic energy of the unloaded cantilever,

$$
E_{\text {strain }} \simeq \frac{1}{2} m_{0} a_{n}^{2} \omega_{n}^{2} \text {. }
$$

From the Rayleigh-Ritz theorem and Eqs. (2)-(4), the resonant frequency of the mass loaded cantilever is approximately

$$
\omega_{n, \Delta m}^{2}=\omega_{n}^{2}\left(1+\frac{\Delta m}{m_{0}} U_{n}^{2}\left(z_{\Delta m}\right)\right)^{-1} .
$$

Using Eq. (5) it is thus possible to calculate the change in resonant frequency of each individual bending mode based on knowledge of the position $z_{\Delta m}$ and mass $\Delta m$ of the attached particle (cell or molecule), the cantilever mass $m_{0}$, and native resonant frequencies $\omega_{n}$.

It is also possible to use the analytical expression in Eq. (5) to determine the mass $\Delta m$ and position $z_{\Delta m}$ of a single attached particle by measuring the resonant frequencies $\omega_{n, \Delta m}$ of the loaded cantilever. The requirement is that the cantilever mass $m_{0}$ and native resonant frequencies $\omega_{n}$ of several bending modes of the unloaded cantilever are known. In order to illustrate that mass and position can be uniquely determined in this manner, it is useful to solve Eq. (5) for the mass ratio and find

$$
\frac{\Delta m}{m_{0}}=\frac{1}{U_{n}^{2}\left(z_{\Delta m}\right)}\left(\frac{\omega_{n}^{2}}{\omega_{n, \Delta m}^{2}}-1\right) .
$$

Now, for a given attached particle, the left hand side of Eq. (6), $\Delta m / m_{0}$, is constant. Likewise in the right hand side of Eq. (6), the position $z_{\Delta m}$ is constant regardless of the bending mode but the values are not known a priori. The frequency ratios $\omega_{n} / \omega_{n, \Delta m}$ are measured and the values change with the bending mode. If the right hand side of Eq. (6) is evaluated for all possible positions of the particle load for each mode given the known values of the frequency ratios $\omega_{n} / \omega_{n, \Delta m}$, the curves for the first four bending modes shown in Fig. 2 result. In Fig. 2, the $y$ axis shows for each of the four modes and assumed position the required mass load to give the measured frequency ratio. Obviously, in the single point where all four curves intersect, the correct unique values of the mass load and position are found. The curves in Fig. 2 are calculated using synthetic data, $\omega_{n} / \omega_{n, \Delta m}$, calculated from Eq. (5) using $\Delta m / m_{0}=1 / 100$ and $z_{\Delta m} / L=0.8$.

The procedure demonstrated above is only useful to show uniqueness, and in a practical measurement analysis, a robust fitting procedure is needed. Solving Eq. (5) for the measured resonant frequencies, we obtain the model for the measured data,

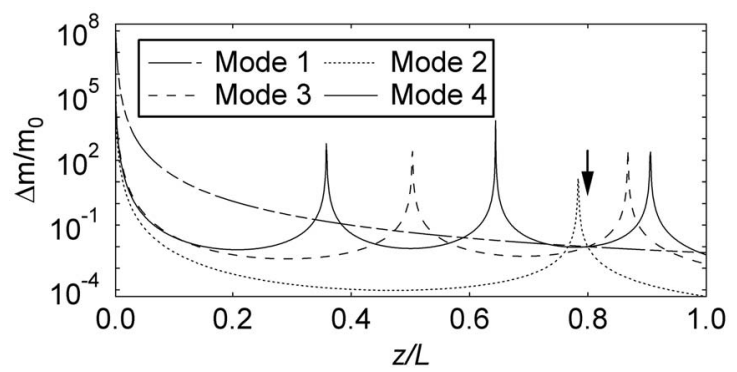

FIG. 2. Calculated mass ratio $\Delta m / m_{0}$ for the first four modes as a function of all possible position, $z / L$. All curves intersect at a single point only $\left(z_{\Delta m} / L=0.8\right.$, marked with an arrow) thereby giving the real position and mass of the attached particle.

$$
\frac{\omega_{n, \Delta m}}{\omega_{n}}=\frac{1}{\sqrt{1+\left(\Delta m / m_{0}\right) U_{n}^{2}\left(z_{\Delta m}\right)}},
$$

with the unknown mass and position as parameters, and from which they can be determined by iteration. Thus, if $N$ modes, $n=1,2, \ldots, N$, are measured and the measurement errors are assumed to have a normal distribution, we minimize

$$
\chi^{2}=\sum_{n=1}^{N} \frac{1}{\sigma_{n}^{2}}\left(\frac{\omega_{n, \Delta m}}{\omega_{n}}-\frac{1}{\sqrt{1+\left(\Delta m / m_{0}\right) U_{n}^{2}\left(z_{\Delta m}\right)}}\right)^{2},
$$

where $\sigma_{n}^{2}$ is the variance of the measured frequency ratio $\omega_{n, \Delta m} / \omega_{n}$. The error estimates for the extracted mass ratio $\Delta m / m_{0}$ and position $z_{\Delta m}$ are calculated using the algorithm. ${ }^{12}$

The mass of the cantilever must be known in order to estimate the mass of the attached particle. The cantilever mass can be calculated from parameters of the materials used in fabrication and the designed or measured geometrical dimensions with an uncertainty of a few percent or less. This error on the actual cantilever mass directly affects the accuracy of the calculated mass of the attached particle and could well be the dominant contribution to the total mass error, but the error is small enough to be acceptable in most cases.

To verify the theoretical findings, data from previously reported experiments on a micrometer sized cantilever have been used..$^{8}$ The cantilever length, width, and thickness were approximately 153,11 , and $1.05 \mu \mathrm{m}$, respectively; the cantilever was fabricated in $\mathrm{SiO}_{2}$ with a $\mathrm{Au} / \mathrm{Ti}(100 \mathrm{~nm} / 10 \mathrm{~nm})$ coating on the topside; thus the estimated total mass of the cantilever is $m_{0} \simeq 7 \mathrm{ng}$. In the experiments a single gold bead with a radius of $0.9 \mu \mathrm{m}$, corresponding to a mass of approximately $60 \mathrm{pg}$, is positioned and manipulated on the cantilever. The position of the gold bead is determined from optical images with an estimated accuracy of $\pm 1 \mu \mathrm{m}$. For each position of the gold bead, the change in resonant frequency was recorded for the first four bending modes while the cantilever was resonating in a low ambient pressure of 0.5 mbar to ensure a high quality factor for the resonator and good resonant frequency resolution.

The measured unloaded resonant frequencies for the first four bending modes of the cantilever were $\omega_{n} /(2 \pi)$ $=30.7,192.5,539.1,1056.5 \mathrm{kHz}$. Figure 3 shows measured relative changes in resonant frequency as a function of the optically measured position of the gold bead; the relative frequency changes calculated from Eq. (5) are shown for comparison. Obviously, theory and experiments are in good agreement. 


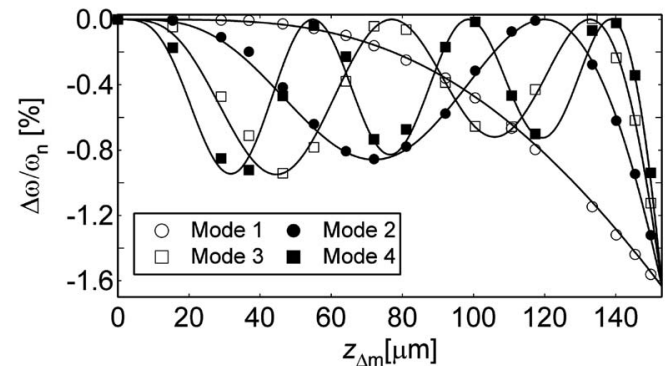

FIG. 3. Measured relative resonant frequency change $\Delta \omega_{n} / \omega_{n}$ of the first four modes as a function of the position of the attached gold bead (symbols). The curves show the calculated relative resonant frequency changes according to Eq. (5).

The extracted gold-bead mass ratio $\Delta m / m_{0}$ and position $z_{\text {freq }}$ obtained using the fitting procedure and the measured resonant frequencies are shown in Fig. 4 as a function of the visually determined bead position $z_{\mathrm{opt}}$. The extracted positions are seen to agree very well with the visually determined positions almost within the estimated errors on these, except for the single point close to the cantilever base where a significant disagreement is seen. At this point the fit returns a false position (and mass) probably due to the very small frequency changes measured at this position and due to inherent shortcomings in the model. First, near the base, the assumption of zero lateral stress becomes invalid. Second, any deviation from a perfectly clamped condition of the beam base will affect the region closer to the base the most. Finally, close to the base of the cantilever, $U_{n}^{2}(z) \simeq A_{n}^{2} \kappa_{n}^{4} z^{4}$ $+O\left(z^{5}\right)$; thus extracted values of $\Delta m / m_{0}$ and $z$ are correlated according to $z^{4} \times \Delta m / m_{0}=$ const independent of the actual fitting procedure used, and the resulting equations used to extract the two parameters become linearly dependent. This problem persists until the bead is placed close to the first antinode of the highest mode measured (here mode 4 at $z / L \sim 0.2)$. Actually, the $\chi^{2}$ surface for this measurement point has a local minimum near the correct position and mass, but due to the correlation and the particular distribu-

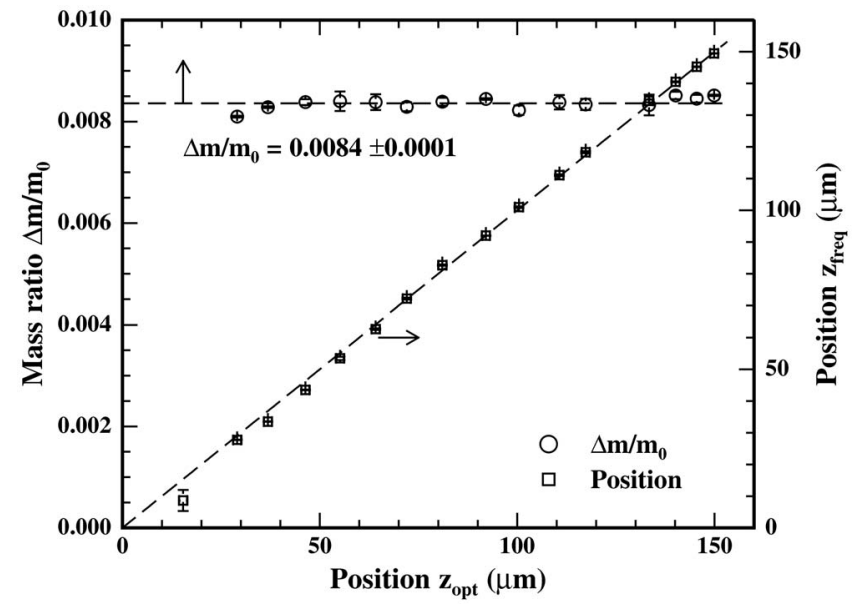

FIG. 4. The position $z_{\text {freq }}$ and mass ratio $\Delta m / m_{0}$ of the attached gold bead extracted from resonant frequency measurements as a function of the visually determined position $z_{\mathrm{opt}}$. tion of measurement errors, there is a global minimum at the reported erroneous position and mass. Apparently, except for this single point, the accuracy of the extracted positions seems to exceed the accuracy of the visually determined positions.

The average extracted mass ratio is $\Delta m / m_{0}$ $=0.0084 \pm 0.0001$ when the point closest to the cantilever base is omitted. Thus, the calculated mass of the gold bead is

$$
m_{\text {bead }}=\Delta m=7 \mathrm{ng} \times(0.0084 \pm 0.0001) \simeq 59 \pm 1 \mathrm{pg},
$$

which is in perfect agreement with the expected value.

The almost perfect agreement between the extracted mass and the expected value when the mass is positioned on the outer $4 / 5$ of the cantilever clearly demonstrates the possibility to do mass distribution analysis without prior knowledge about or control over the position of the added particle.

The method could very well be applied to single-cell measurements or nanoparticle detection, where a very accurate particle mass measurement is needed and a time consuming position measurement using scanning electron microscopy imaging is inconvenient. It could equally well be used for determining the mass of cells or particles flowing inside a cantilever as demonstrated by Burg and Manalis. ${ }^{9}$

The method could also prove very useful for enhancing the functionality of cantilever based mass sensors. This could be achieved by having several areas on the cantilevers coated for sensing of specific and different target molecules. By measuring several vibration modes during operation, the binding of desired targets in one area could be differentiated from binding in other areas. In principle, this would make it possible to design an artificial nose $\mathrm{e}^{13,14}$ using only a single cantilever.

We want to thank Dr. F. Amiot for fruitful discussions. CINF is sponsored by The Danish National Research Foundation.

${ }^{1}$ M. Roukes, Phys. World 14(2), 25 (2001).

${ }^{2}$ G. Y. Chen, T. Thundat, E. A. Wachter, and R. J. Warmack, J. Appl. Phys. 77, 3618 (1995).

${ }^{3}$ T. Thundat, E. A. Wachter, S. L. Sharp, and R. J. Warmack, Appl. Phys. Lett. 66, 1695 (1995).

${ }^{4}$ K. L. Ekinci, X. M. H. Huang, and M. L. Roukes, Appl. Phys. Lett. 84, 4469 (2004).

${ }^{5}$ Y. T. Yang, C. Callegari, X. L. Feng, K. L. Ekinci, and M. L. Roukes, Nano Lett. 6, 583 (2006).

${ }^{6}$ M. L. Roukes, International Workshop on Nanomechanical Sensors, 2006 (unpublished).

${ }^{7}$ J. Teva, G. Abadal, F. Torres, J. Verd, F. Perez-Murano, and N. Barniol, Ultramicroscopy 106, 808 (2006).

${ }^{8}$ S. Dohn, R. Sandberg, W. Svendsen, and A. Boisen, Appl. Phys. Lett. 86, 233501 (2005).

${ }^{9}$ T. P. Burg and S. R. Manalis, Appl. Phys. Lett. 83, 2698 (2003).

${ }^{10}$ A. N. Cleland, Foundations of Nanomechanics (Springer, Berlin, 2003).

${ }^{11}$ S. D. Senturia, Microsystem Design, 4th ed. (Kluwer Academic, Dordrecht, 2002), Chap. 10, pp. 239-265.

${ }^{12}$ P. R. Bevington and D. K. Robinson, Data Reduction and Error Analysis for the Physical Sciences (McGraw-Hill, New York, 1992), Chap. 11.

${ }^{13}$ H. P. Lang, M. K. Baller, R. Berger, C. Gerber, J. K. Gimzewski, F. M. Battiston, P. Fornaro, J. P. Ramseyer, E. Meyer, and H. J. Guntherodt, Anal. Chim. Acta 393, 59 (1999).

${ }^{14}$ M. K. Baller, H. P. Lang, J. Fritz, C. Gerber, J. K. Gimzewski, U. Drechsler, H. Rothuizen, M. Despont, P. Vettiger, F. M. Battiston et al., Ultramicroscopy 82, 1 (2000). 